\title{
How do we spell maze (2.0)? A dialogue concerning definition and outcome
}

\author{
Niv Ad, MD
}

From the Department of Cardiovascular and Thoracic Surgery, West Virginia University, Morgantown, WVa. Disclosures: N.A. is a consultant for Medtronic, a member of the speaker's bureau for AtriCure, proctor and member of the speaker's bureau for LivaNova, on the advisory board for Nido Surgical, and co-owner for Left Atrial Appendage Occlusion, LLC.

Received for publication July 16, 2018; accepted for publication July 21, 2018; available ahead of print Aug 16, 2018 .

Address for reprints: Niv Ad, MD, Department of Cardiovascular and Thoracic Surgery, West Virginia University, 1 Medical Center Dr, Morgantown, WV 26506 (E-mail: nivadmd14@gmail.com).

J Thorac Cardiovasc Surg 2019;157:246-7

$0022-5223 / \$ 36.00$

Copyright (c) 2018 by The American Association for Thoracic Surgery

https://doi.org/10.1016/j.jtcvs.2018.07.031

Atrial fibrillation (AF) is common in patients undergoing mitral valve surgery, with approximately $30 \%$ of patients in AF at the time of surgery. ${ }^{1}$ Multiple studies have demonstrated that AF is associated with increased complications and decreased survival in these patients. Concomitant surgical ablation at the time of mitral valve surgery was granted a Level A class 1 indication with credible evidence on shortand long-term efficacy and safety.,3 The Cardiothoracic Surgical Trials Network prospective randomized trial ${ }^{4}$ is the largest of its kind in this field. The results of the trial raised some intense discussions and debates related to the comparison of the efficacy of pulmonary vein isolation and biatrial lesion set. ${ }^{4}$ The somewhat charged debate overshadowed, to some extent, the important findings of the trial that demonstrated that surgical ablation at the time of mitral valve surgery resulted in a significantly higher percentage of patients in sinus rhythm at 6 and 12 months compared with those who were left untreated. Therefore, the contribution of the trial to the field is not debatable.

Cardiac surgeons, referring cardiologists, and electrophysiologists should be familiar with the results of the trial; however, several important facts about the trial should be approached with more scrutiny.

1. The study protocol allowed freedom in the choice of surgical ablation technology and devices. The decision that allowed the use of unipolar radiofrequency pen that is known to be inconsistent in its application of transmural lesions is problematic. ${ }^{2,5}$ What should surgeons do with this information? When looking at the results, one should be aware that some of the lesions that were applied with the unipolar pen may not be consistent or transmural because of the inherent limitation of the device and the mode of energy delivery. Therefore, any comparisons between lesion set may not be valid because the variability between surgeons and devices may have driven the outcome rather than the lesion pattern.

2. Many of the patients in this trial had more than mitral valve surgery only; therefore, the conclusion of the trial

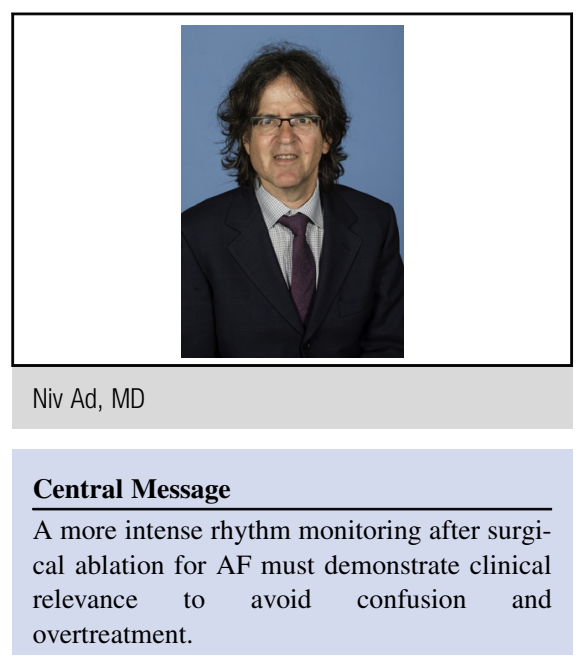

See Article page 234 . may not be generalized to concomitant surgical ablation and isolated mitral valve procedures.

3. A common misconception related to the Cox maze III/IV procedure was noted in this trial, namely, that the procedure is not effective in treating typical flutter. However, the Cox maze III/IV procedure includes 2 lesions to the tricuspid valve annulus, and therefore is very effective in treating atrial flutter. According to the study protocol, surgeons were allowed to perform an extra ablation line to the tricuspid valve (cavo-isthmus line, a third tricuspid annular lesion) in patients with a history of atrial flutter. This extra lesion is unnecessary and most likely accounts for the high rate of pacemakers reported. The readers should acknowledge that the authors referred to the procedures as biatrial maze procedure and not biatrial Cox maze III/IV procedure most probably because of this variation in the lesion set.

By using data generated from patients enrolled in the Cardiothoracic Surgical Trials Network trial, ${ }^{6}$ the work by Blackstone and colleagues published in this issue of the Journal is a notable effort to establish a new analytic approach to define rhythm end points after surgical ablation for AF. The authors' intentions are clearly focused on improving the reliability of the rhythm status capture and assessment after surgical ablation. The statistical work and careful scientific analysis are to be recognized and applauded. Their working theory is based on the notion that the more you monitor the more you find, which is acceptable across all aspects of 
electrophysiology. In this study, it was shown that by using a 3-lead transtelephonic monitor, with strips transmitted weekly, the prevalence and the load of atrial arrhythmia could be captured reliably and in a reviewable fashion. The data were then used to compare the potential effect of the lesion set on the rhythm status outcome using the original study patient groups. The study findings suggest that the biatrial lesion set is associated with a lower prevalence of recurrent atrial arrhythmia. These findings are somewhat different than the original report that suggested no apparent advantage of a biatrial lesion set over pulmonary vein isolation only. The new findings are more in line with many previous and credible reports demonstrating that the biatrial lesion is superior to the left atrial only lesion set. ${ }^{7}$ This impressive theory and analysis should be further tested so we can compare it with the benchmark as agreed and summarized in the 2017 Heart Rhythm Society guidelines. ${ }^{8}$ Currently, we should all adhere to the guidelines as a representation of the consensus that allow us to compare outcomes across procedures and different types of clinical scenarios.

The critical questions that we all have to ask ourselves should be focused around what we refer to as cost benefit. It is clear that more monitoring can be helpful in better understanding the magnitude of potential recurrence of atrial arrhythmia. However, our experience and that of others have shown that there are some significant hurdles to accomplish this mission.

1. The cost: It is currently unclear what would be the position of the insurance companies in supporting the cost of the devices and the extensive resources required for reading and interpreting the results.

2. The compliance of the patients is going to be a challenge. Even in this well-controlled trial, the number of patients not participating in the transtelephonic monitor program, either at all or regularly, was significant.

3. The potential medical liability arises, because the results need to be read when transmitted to avoid misdiagnosis and delays in treatment. This will require a special infrastructure, and there is an expected increase in cost. Maybe the most important aspect of this analytic technique is whether it carries a significant clinical relevance. There are several reports from leading centers that demonstrated a high rate of freedom from atrial arrhythmia, freedom from embolic strokes, and improved quality of life using scheduled Holter monitoring only. This type of practice is being challenged by the authors for the right reasons. In general, we all agree that especially during the first 12 months after the procedure, a more intense monitoring may result in higher rates of detection of recurrent arrhythmia. Nevertheless, although atrial arrhythmia can be asymptomatic, the likelihood of multiple embolic events and strokes to be with no clinical consequences is extremely low. To date, there are multiple publications involving thousands of patients in whom rhythm status reports are based on Holter monitoring only for patients after the Cox maze procedure. These reports all share a high success rate and a low stroke rate, with many of the patients free of oral anticoagulation. If we agree that strokes are associated with atrial arrhythmia, then we should probably acknowledge the fact that periodic Holter monitoring in asymptomatic patients is fairly reliable. Otherwise, we cannot consistently explain the low incidence of strokes. ${ }^{9}$

This new analytic approach should be tested against the benchmark, not only for arrhythmia events but also in clinical outcomes that include thromboembolic events, the need for anticoagulation, antiarrhythmic drugs, and quality of life. Therefore, the next study to validate this new method should include, as its primary end point, not only a comparison of the rhythm status off and on antiarrhythmic medication but also a comparison of the potential impact on clinical outcomes in terms of the quality of life, freedom from stroke (potentially better targeting treatment of silent atrial arrhythmia), and longevity of the patient. Without the ability to show a positive clinical relevance, we are running the risk of entering into a state of info-obesity, where we have too much information with not much use for it, a situation that may unfortunately cause confusion and may even risk some patients with unnecessary treatment.

In summary, our field is much stronger and better with this newly presented and exciting method. The authors ${ }^{6}$ should use their clinical and scientific expertise to associate this approach with improved clinical outcomes after surgical ablation for AF.

\section{References}

1. Rankin JS, Grau-Sepulveda MV, Ad N, Damiano RJ Jr, Gillinov AM, Brennan JM, et al. Association between surgical ablation and operative mortality after mitral valve surgery. Ann Thorac Surg. 2018;105:1790-6.

2. Ad N, Damiano RJ Jr, Badhwar V, Calkins H, La Meir M, Nitta T, et al. Exper consensus guidelines: examining surgical ablation for atrial fibrillation. J Thorac Cardiovasc Surg. 2017;153:1330-54.

3. Badhwar V, Rankin JS, Damiano RJ Jr, Gillinov AM, Bakaeen FG, Edgerton JR, et al. The Society of Thoracic Surgeons 2017 Clinical Practice Guidelines for the Surgical Treatment of Atrial Fibrillation. Ann Thorac Surg. 2017;103:329-41.

4. Gillinov AM, Gelijns AC, Parides MK, DeRose JJ Jr, Moskowitz AJ, Voisine P, et al; CTSN Investigators. Surgical ablation of atrial fibrillation during mitral-valve surgery. N Engl J Med. 2015;372:1399-409.

5. Damiano RJ Jr, Badhwar V, Acker MA, Veeragandham RS, Kress DC, Robertson JO, et al. The CURE-AF trial: a prospective, multicenter trial of irrigated radiofrequency ablation for the treatment of persistent atrial fibrillation during concomitant cardiac surgery. Heart Rhythm. 2014;11:39-45.

6. Blackstone EH, Chang HL, Rajeswaran J, Parides MK, Ishwaran H, Li L, et al Biatrial maze procedure versus pulmonary vein isolation for atrial fibrillation during mitral valve surgery: new analytical approaches and end points. J Thorac Cardiovasc Surg. 2019;157:234-43.e9.

7. Barnett SD, Ad N. Surgical ablation as treatment for the elimination of atrial fibrillation: a meta-analysis. J Thorac Cardiovasc Surg. 2006;131:1029-35.

8. Calkins H, Hindricks G, Cappato R, Kim YH, Saad EB, Aguinaga L, et al. 2017 HRS/EHRA/ECAS/APHRS/SOLAECE expert consensus statement on catheter and surgical ablation of atrial fibrillation. Heart Rhythm. 2017;14:e275-444.

9. Ad N, Holmes SD, Massimiano PS, Rongione AJ, Fornaresio LM. Long-term outcome following concomitant mitral valve surgery and Cox maze procedure for atrial fibrillation. J Thorac Cardiovasc Surg. 2018;155:983-94. 